\title{
Sur la coagulation de l'hémolymphe chez Locusta migratoria
}

\author{
par M. BREHELIN \\ Laboratoire de Biologie générale, Faculté des Sciences, \\ 12 , rue de l'Université, F 67000 Strasbourg
}

Chez Locusta migratoria, la coagulation de l'hémolymphe consiste en une gélification plasmatique sans agglomération des cellules (type I de Grégoire) (Grégoire et Florkin, 1950). De nombreuses observations en microscopie optique et en microscopie électronique ont montré qu'un seul des types hémocytaires, les coagulocytes, présentent lors du prélèvement de l'hémolymphe des transformations qui induisent la coagulation du plasma. Cependant, très peu d'études ont été menées à bien en ce qui concerne les phénomènes biochimiques de la coagulation (Grégoire, 1974). On ignore, en particulier, quelles sont les protéines plasmatiques qui forment le coagulum, ainsi que leur proportion par rapport à la protéinémie totale de l'hémolymphe. De même, le «principe actif » qui induit la coagulation plasmatique n'a jamais pu être isolé des hémocytes ni d'aucun autre tissu de l'insecte.

Notre travail porte sur ces deux points : étude des protéines plasmatiques et étude des facteurs cellulaires.

L'analyse électrophorétique du plasma (hémolymphe sans hémocytes) et du sérum (surnageant clair obtenu après coagulation et centrifugation de l'émolymphe) de larves de stade $\mathrm{V}$ de Locusta, nous a permis de montrer que le plasma contient une (ou un petit nombre) de protéine qui précipite lors de la coagulation pour former le coagulum. Cette protéine est une glyco-lipo-protéine de PM compris entre 500000 et 1 million. A certains jours du stade larvaire $\mathrm{V}$, elle représente environ $20 \%$ dé la protéinémie totale de l'hémolymphe, soit $7 \mathrm{~g} / 1$.

La vitesse de coagulation de l'hémolymphe de larves, du cinquième stade de Locusta migratoria, varie dans le même sens que le nombre de coagulocytes par unité de volume ; cette observation a été faite en étudiant les variations normales de l'image sanguine et également en provoquant expérimentalement des modifications du nombre des hémocytes circulant. 
Nous avons pu isoler à partir des cellules sanguines un facteur soluble qui provoque la coagulation du plasma; les analyses électrophorétiques ont montré que cette coagulation affecte la même bande protéique que celle qui précipite lors d'une coagulation normale. Ce facteur cellulaire est thermolabile $\left(65^{\circ}\right.$ pendant $\left.10 \mathrm{mn}\right)$ et non dialysable. Il ne peut être isolé, au moins sous sa forme active, des granulocytes seuls ni d'autres tissus de l'insecte (muscle, corps gras, hypoderme).

\section{Conclusion.}

Deux types de facteurs intervenant dans la coagulation ont été isolés de l'hémolymphe de Locusta migratoria : des facteurs plasmatiques (protéines du coagulum) et des facteurs hémocytaires qui induisent la coagulation plasmatique. La suite de notre travail portera sur la purification de ces facteurs et leur analyse chimique.

\section{Bibliographie}

Gregoire (C.) et Florkin (M.), 1950. - Blood coagulation in arthropods. I. The coagulation of insect blood, as studied with the phase contrast microscope. Physiol. comp. Ecol., 2, 126-139.

Gregorre (C.), 1974. - Hemolymph coagulation, in: The Physiology of Insecta, vol. $V$, 309-360, Academic Press, édit., London. 